\title{
Diagnostic and Therapeutic Implications of Conjoined Nerve Root Anomalies: A Senegalese Study of Three Cases
}

\author{
Yannick Canton Kessely ${ }^{1 *}$, Ibrahima Tine ${ }^{2}$, Maguette Gaye Sakho ${ }^{3}$, Maguette Mbaye ${ }^{4}$, Mahamoud \\ Ali Meidal ${ }^{5}$, Youssou Traore ${ }^{6}$, Abdoulazize Diop ${ }^{7}$, Youssoupha Sakho ${ }^{8}$
}

\author{
${ }^{1} \mathrm{MD}$, Neurosurgeon, Grand Yoff Hospital, Dakar, Senegal \\ ${ }^{2} \mathrm{MD}$, Neurosurgeon, Princioal Hosoital, Dakar, Senegal \\ ${ }^{3} \mathrm{MD}$, Neurosurgeon, Assistant Professor, Grand Yoff Hospital, Dakar, Senegal \\ ${ }^{4} \mathrm{MD}$, Neurosurgeon, Assistant Professor, Fann Hospital, Dakar, Senegal \\ ${ }^{5} \mathrm{MD}$, Neurosurgeon, Fann Hospital, Dakar, Senegal \\ ${ }^{6} \mathrm{MD}$, Neurosurgeon, Fann Hospital, Dakar, Senegal \\ ${ }^{7} \mathrm{MD}$, Professor of Neurosurgery, Head of Service of Neurosurgery, Department of Princioal Hosoital, Dakar, Senegal \\ ${ }^{8} \mathrm{MD}$, Professor of Neurosurgery, Head of Service of Neurosurgery, Department of Grand Yoff Hosoital \\ * Corresponding Author Address: Neurosurgery Department, Grand-Yoff Hospital, Dakar, Senegal. Tel: 00 221774451378, E-mail: \\ canton_kessely@yahoo.fr
}

Article Type: Case Series

Received: November 21, 2015, Last revised: November 29,2015, Accepted: December 22, 2015

\section{Abstract}

Background \& Aim: Conjoined nerve root is defined as two adjacent nerve roots that share a common dural envelope at some points during their course from the thecal sac. This study reports our experience of conjoined roots involving three cases in Dakar.

Methods \& Materials/Patients: This is a consecutive study from 2013 to 2015 involving patients supported for disc herniation and who have presented conjoined nerve root anomalies.

Results: Three patients aged 32, 35 and 55 including two men have been concerned. Clinical analysis was done on sciatica with neuropathic occurrences in one case and lumbosciatica in two cases. The Lasegue sign was present in two patients at $45^{\circ}$. All three patients benefited a lumbar computerized tomography (CT scan) highlighting a degenerative disc disease with two in L5S1 space and one in L4L5 space. The imaging has not objectified radicular emerging anomalies. MRI objectified only one big root. A surgical root decompression was realized through interlaminar discectomy approach; foraminotomy and full laminectomy enabling diagnosis in intraoperative period. The evolution was favourable in all three cases with full recession of symptomatology.

Conclusion: This study is the first Senegalese series on the lumbo-sciatica by anomaly of root emergence and highlights especially the difficulties for the diagnosis of these anomalies like other sub-Saharan African countries where expansion of MRI for the diagnosis is low, and still very expensive. MRI provides guidance signs and a large root appearance can warn about the existence of these anomalies. A good root release improves the symptoms.

Keywords: Conjoined roots; MRI; Surgery

Please cite this paper as: Kessely Y.C, Tine I, Sakho M.G, Mbaye M, Meidal M. A, Traore Y, Diop A, Sakho Y. Diagnostic and Therapeutic Implications of Conjoined Nerve Root Anomalies: A Senegalese Study of Three Cases. Iran. J. Neurosurg. 2015;1(3):21-25.

\section{Introduction}

Usually operatively found, the anomalies of conjoined roots are defined as two adjacent nerve roots that share a common dural envelope at a point during their course (1). Revealed through a clinical picture of lumbar-radiculalgia, no specific sign related to anomalies has been described. The incidence varies from 2 to $17.3 \%$ in patients that have realized lumbar imaging, and from $8.5 \%$ to $30 \%$ on cadaveric studies (1). The first description was given in 1949 by Zagnoni (2). Known since the myelography era, it is currently benefiting from a better analysis with the contribution of magnetic resonance imaging (MRI). Although it is rare entity, there have been many descriptions regarding it. We report our experience and the management challenge of lumbo-sciatica by roots conjoined anomalies through a study of three patients with a review of literature.

\section{Case Presentation}

From 2013 to 2015, we had a consecutive series at three neurosurgical centers from patients who had surgery in whom clinical diagnosis and radiological investigations concluded to a lumbo-sciatica by herniated disc, but intraoperative findings were in favor of conjoined roots. Three patients were identified.

\section{Results}

\section{Observation No.1}

A 35-year-old man was followed up for right S1 hyperalgesia sciatica evolving for three months and persisting despite medical treatment. Neither notion of trauma nor a past history of lumbago was reported. During diagnosis, an hyperpathic plantar confining the patient to bed was noted with right sciatica 
of type S1 topography without lumbago, with neuropathic occurrences of type, electrical discharges, allodynia and sensation of painful cold. A pain at the Valleix point has been highlighted. A plantar hypoesthesia was found without spinal rigidity or motor deficit. Osteo-tendinous reflexes have not been looked at due to pain, meanwhile a Lasegue sign was found at the end. The spine lumbar imaging has shown a protrusive disc disease in right L5S1 space and an aspect of big root at emerging S1 level (Figure 1). A right L5-S1 interlaminar fenestration permitted assessing the roots moving side-by-side towards lateral recessus (Figure 2): type IIb of Neidre and Mac Nab classification. The medial root was congestive and difficult to mobilize. The lateral recessus was stenosed. These conjoined roots generated space conflict and a foraminotomy without discectomy were achieved. A retrocession of pain occurrences was noted the next day. The patient was discharged from hospital on the 4th day with disappearance of sciatica.

\section{Observation No.2}

A 32-year-old military man was followed up for left lumbosciatica S1 occurring due to sport practice. Clinical diagnosis reveled that he had a $45^{\circ}$ left Lasegue with a 10 centimeter fingerground distance. Patellar and Achilles reflexes were normal. No sensorimotor deficit was detected. The lumbar spine CT scan had highlighted a left compressive herniated disk L5S1 (Figure $3 a)$. The operative indication had been determined after failure of the medical treatment done by analgesic, non-steroidal antiinflammatory, muscle relaxant combined with physiotherapy sessions during 2 months. The operating gesture consisted of a left interlaminar approach. Two nerve roots L5 and S1 with a joint emergence were discovered during intraoperation (Figure 3b). This anomaly corresponds to a Ib type from Neidre et Mac $\mathrm{Nab}$ classification. Roots were studiously repressed towards dural sheath and a discectomy was realized then after with a foraminotomy L5S1 and a medial facetectomy. An immediate scientific post-operative MRI confirmed this root emerging anomaly (Figure 4).

\section{Observation No.3}

This is about a 55-year-old lady, a housewife suffering from lumbar-radiculalgia for over 3 years and the history of goiter surgery two years earlier, under Levothyrox, who came to check up for right hyperalgesia lumbar-radiculalgia L4-L5. Clinical observation found a L4-L5 motor deficit associated with hypoesthesia, $\mathrm{a} 45^{\circ}$ right Lasegue with a lumbar spinal syndrome. The CT scan of lumbar spine objectified a degenerative disc L4-L5 with a root conflict (Figure 5). After a well-conducted medical treatment without improvement, a surgery indication was decided and involved to a right unilateral discectomy by surgical interlaminar approach. Due to symptomatology persistency and identification of a foreign-body in the surgical site during scanner control, a second surgery was decided and was realized within an interval of 72 hours. This gesture consisted to the realization of a full laminectomy highlighting two converging adjacent roots and that fuse (Figure 6). The discectomia was done with satisfying post-operational effects.
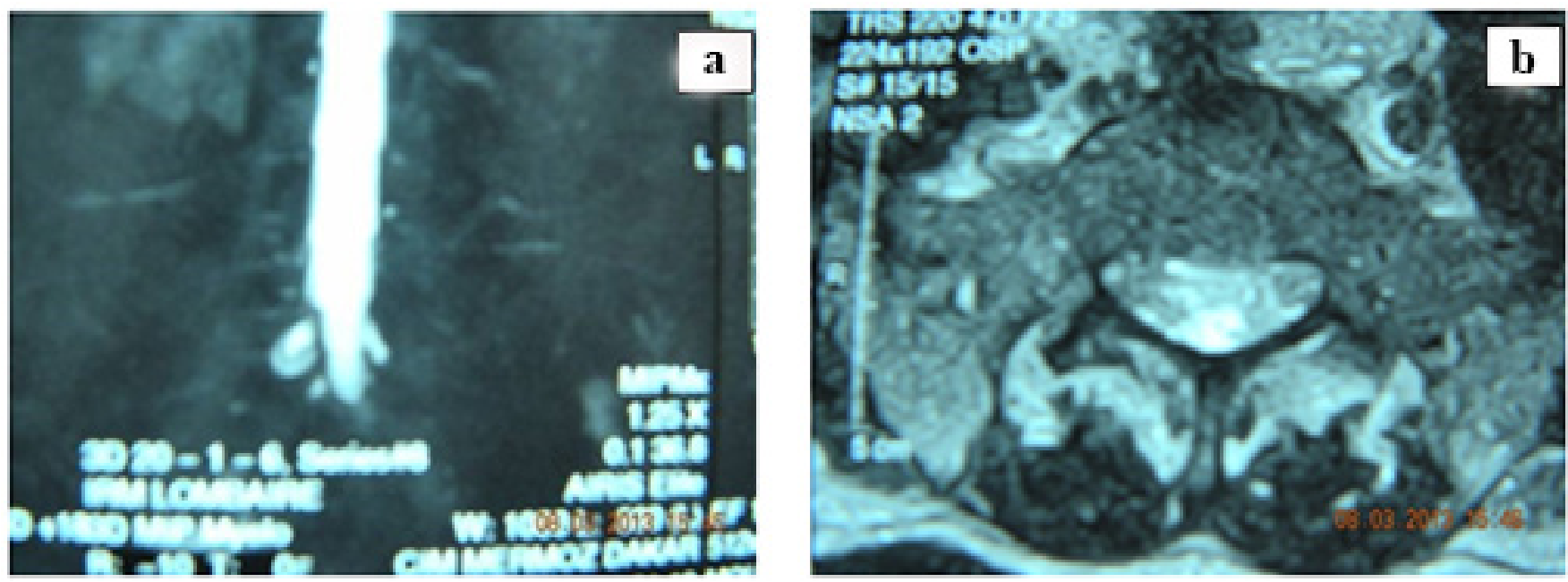

Figure 1. a) Lumbar T2 sequence MRI images in coronal section showing a big root at the right. b) Lumbar T2 sequence MRI in sagittal section showing a protrusion disc in L5S1
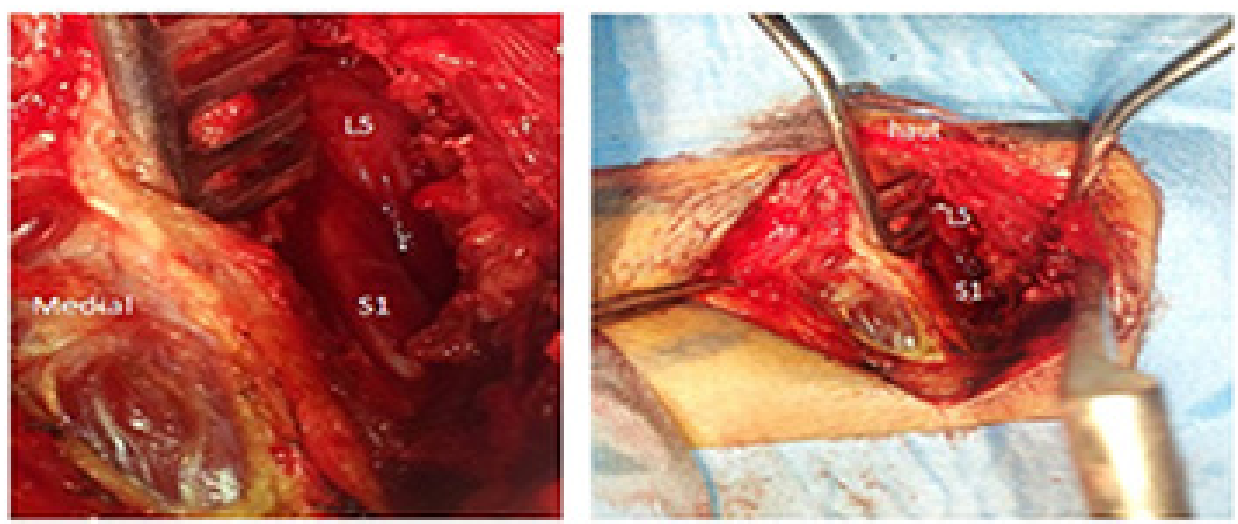

Figure 2. Intraoperative view. Type IIb of Neidre and Mac Nab Classification 

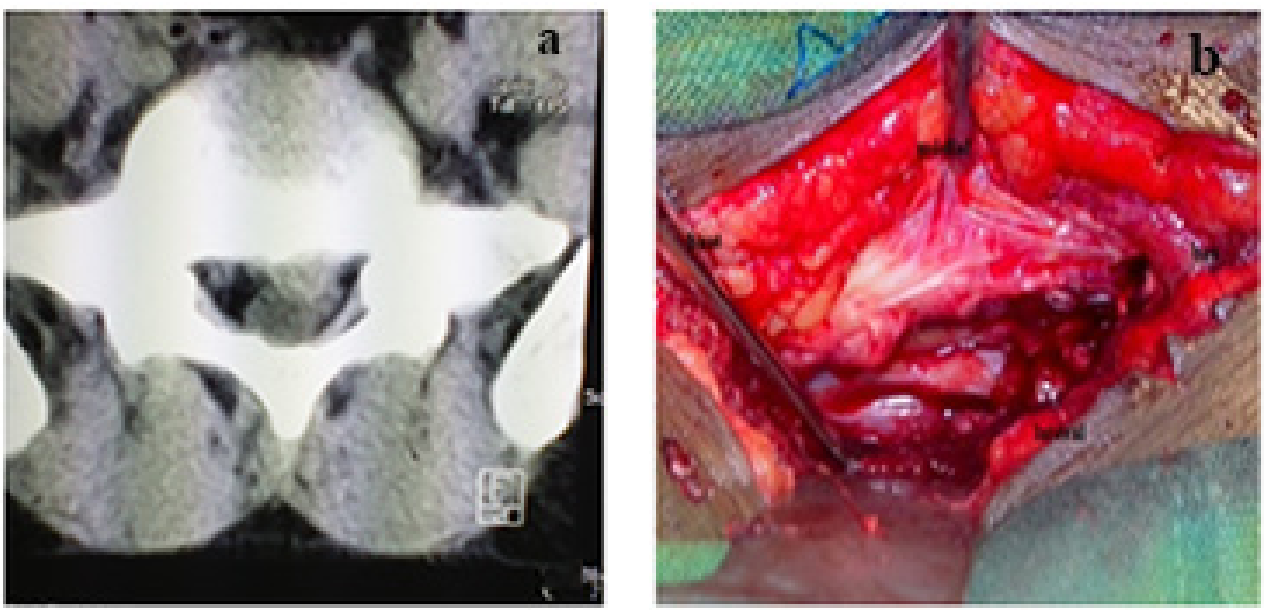

Figure 3. a) CT lumbar spine showing left postero-lateral disc herniation by L5S1, 3. b) Intraoperative view. Type Ib of Neidre and MacNab Classification
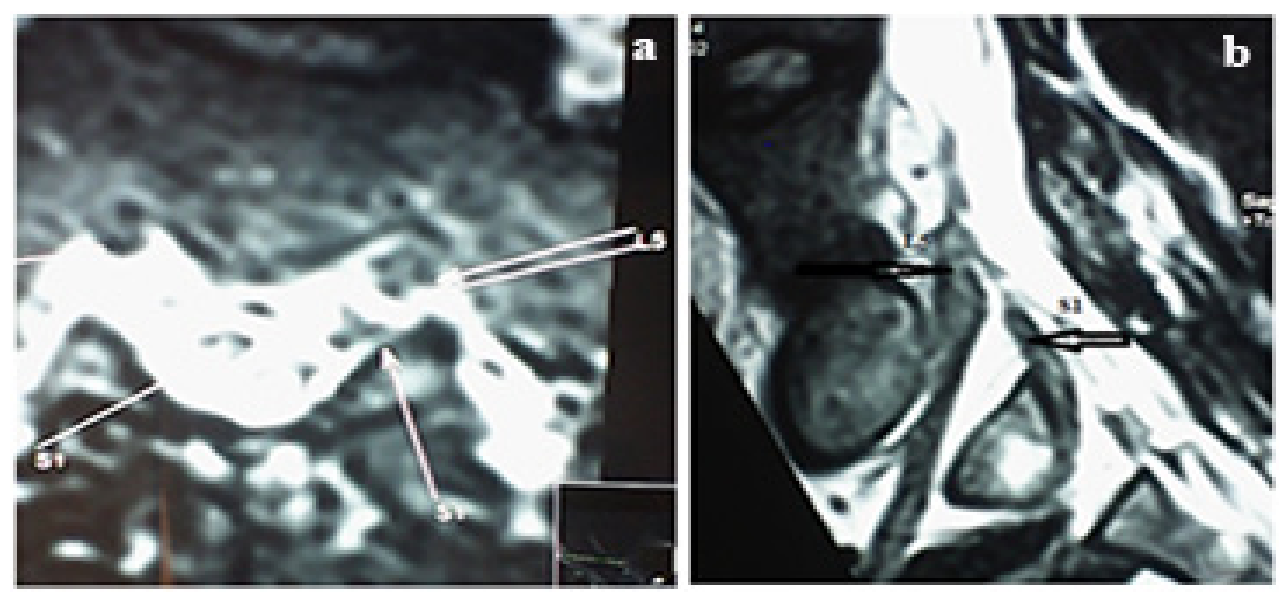

Figure 4. Post operative lumbar T2 sequence MRI. Axial section a) and sagittal on b) highligthing the L5S1 roots
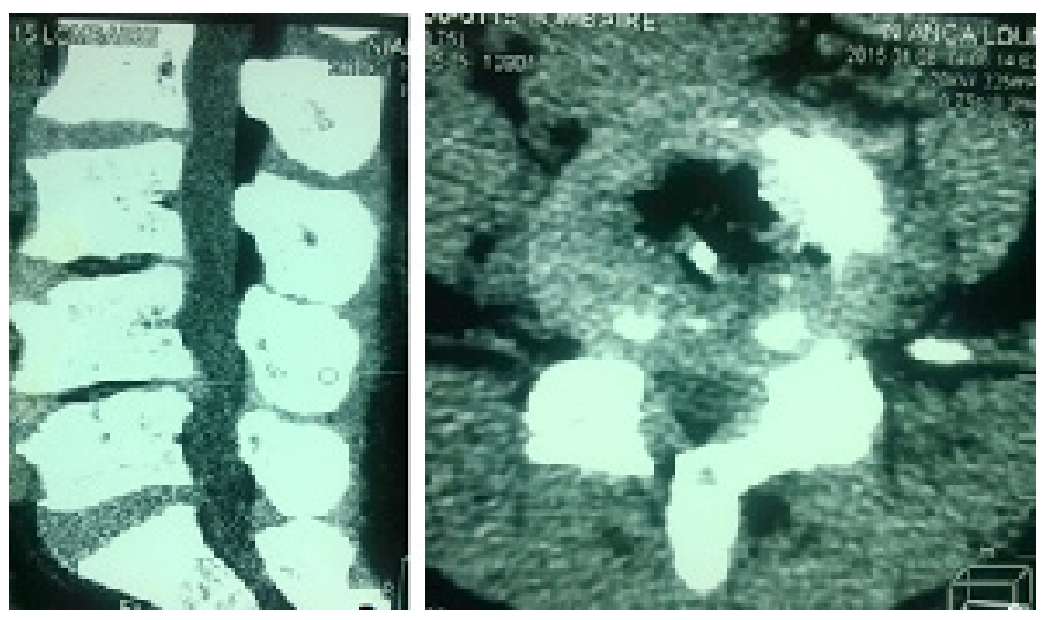

Figure 5. CT-scan of the lumbar spine: degenerative disc disease L4-L5 

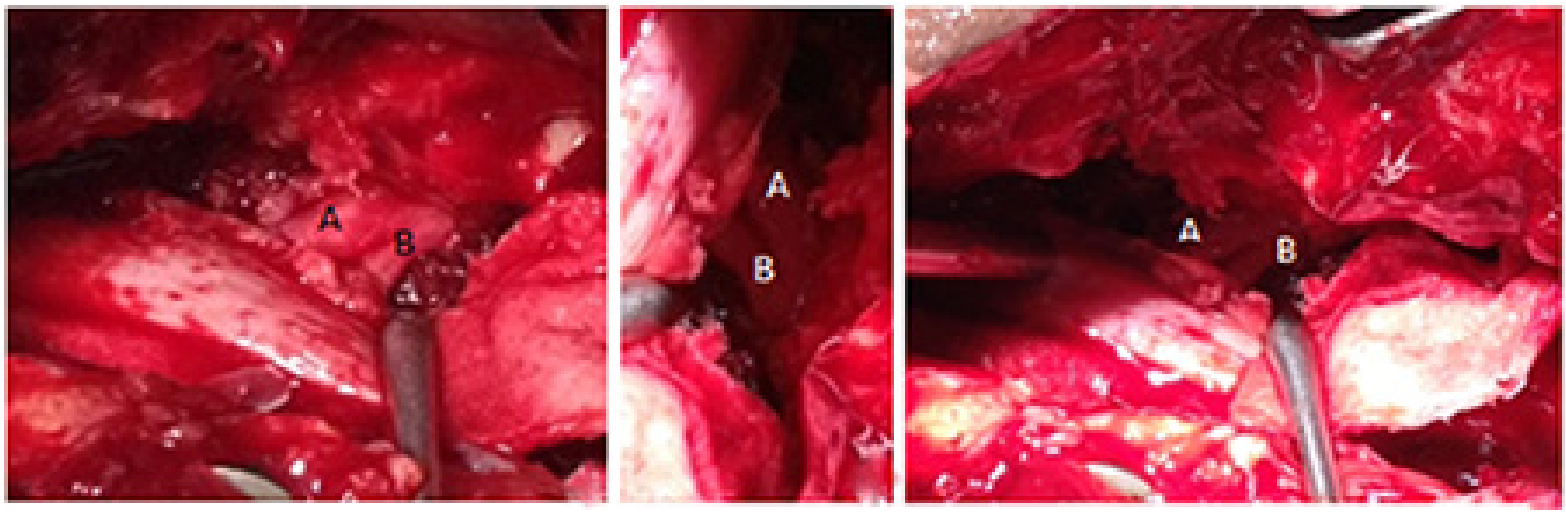

Figure 6. Intraoperative view highlighting conjoined roots. Unclassified type

\section{Discussion}

Between 2013 and 2015, we have listed three patients aged respectively 32,35 and 55 years including two men and one woman. Gender predominance and age predilection are not established most of the time. Taghipour et al. (2009) also reported a slight male predominance $(59.1 \%)$ on their collected series (22 patients) in 11 years (3). Nerve roots anomalies constitute a relatively rare affection where incidence was diversely reported. And so, White et al. (1982) found a $1.3 \%$ incidence during 25 years of exercising disc surgery (4). During the study period, we reported 613 surgical interventions for degenerative spine, what makes us an incidence of $0.49 \%$. This testifies the rarity of this entity. Scanning studies reported weak incidence varying form $2 \%$ against $2.2 \%$ at myelography $(5,6)$. This corroborates the case of our patients all of whom had a CT-scan of the lumbar spine but whose diagnosis of joint root has been mentioned in any of them . High incidences from $14 \%$ are only reported on series regarding cadaveric studies (6). We do not report autopsy discovery because the autopsy is not a common practice for cultural considerations. Intraoperative diagnosis is the most frequent mode of discovery. Embryology of nerve roots anomaly still remains uncertain (7). Conjoined roots are mostly unilateral, even in a bilateral surgery exploration cases. Helms et al. (1982) during their study described a bilateral case on five patients and Taghipour et al. (2009) a case on 22 patients (8,3). Phillips (1993) showed that posterior roots are more concerned by the anomaly and the cause is still unknown (9). Since the first description in 1949 by Zagnoni, several classifications related to this pathology have been proposed. The complexity of clinical forms leads to the multiplicity of classifications. Our three (3) patients were of type IIb and Ib of Neidre of MacNab classification, respectively. The anomaly presented by the third patient has been reported in any: it involves the fusion of two roots, cranial and caudal ipsilateral, preceding the intervertebral foramina emergence. The anomaly was on roots L5 and S1 in the first case at right, and in the second case at left; for the third patient, it was about right L4 and L5 roots. From clinical point of view, nerve roots anomalies and malformations are not symptomatic according to some authors, but it is the adjunction of degenerative occurrences that are responsible for a compression such as herniated disc, a stenosis of lateral recessus or spondylolisthesis (1), which can make them symptomatic. This corresponds to the chart evidence showed by our three patients, among whom one was not symptomatic until his sports accident. Furthermore, all of the 22 patients comprising in the series of Taghipour et al. (2009) showed a disc protrusion as underlying pathology with $90 \%$ lateralized. However, other authors (10) argue that symptomatic character can exist outside any compression mechanism and result in an intermittent, bad systematized and sometimes bi-radicular. Clinical signs in this case may be generally dominated by claudication such as prodrome with or without radiculalgia. According to Trimba (7), lumbago is the frequently sign found during radicular anomalies. As for Lotan (2010), radiculalgia at rest even, occurs more frequently in people/patients with hernitia disc. That was confirmed by the state of our patient (No.2) who had herniated disc in L5S1. We think that this patient's neuropathic pain may be caused through friction of one of roots contained in lateral recessus confined during an abrupt movement involving lumbar spine. The sciatica intermittent character and the lack of Lasegue sign may guide diagnosis; but the presence of Lasegue sign is not unanimous. A Lasegue at the end of the process was objectified in one of our patients, the other two were a Lasègue at $45^{\circ}$. Furthermore, some authors have reported cases of root anomalies in patients having symptomatology of disc herniation but in intraoperation did not present any disc herniation (11). According to Decq et al. (1989), symptomatology of these anomalies would be linked to limited mobility and not necessarily to a compressive element (12). Diagnosis is usually done during intraoperative period. According to Lotan et al. (2010), diagnosis by myelography, by CT scan or by preoperative MRI remains a challenge despite the improvement of investigating means (1). The spine lumbar CT scan of our patients did not allow thinking of conjoined root, they suggested a degenerative disc disease. However, for Younes (2009), although the scan is less contributing to the diagnosis, it can sometimes show the anomaly in a form of big root, bilobate root or duplicated at the level of lateral recessus (13). This aspect of a big root was objectified on patient No.1 MRI. Bone changes can be noted in association with conjoined nerve roots. Some (8) do report on enlargement of lateral recessus from malformation side, what is not observed in case of hernia exclusion. At myeloscanner, confusions are possible between the root and disc fragment (14). Currently, MRI is the exam of choice to objectify the different types of anomaly even those whose diagnosis is difficult to do at myeloscanner, particularly in coronal MRI images, sequences T1 and T2. For patient No.1, MRI showed a big root aspect rather. Despite the description of these different signs, many false positives are possible. MRI in post-operative period of patient No.2, highlighted the proximity of L5S1 roots. Pre-operative diagnosis is a real challenge. The use of computed tomography for the diagnosis of the discopathy is very widespread in sub-Saharan Africa. However MRI introduced since 2007 in Senegal remains with limited accessibility because of cost (four times the guaranteed minimum wage) and availability perspective. This can be explained by the fact that only one of the three patients received pre-operative MRI having found one 
aspect of big root. Note also that pre-operative discoveries in the literature are not also easy.

The aim of surgery is to provide a root release (foraminotomy, arthrectomy or pediculectomy) and an eventual resection of associated hernia (13). Asymptomatic cases or of incidental findings do not necessarily need treatment (15). As for Jeffrey et al. (1988), a conservative approach with corticoid infiltration is recommended for anomaly cases without disc herniation but symptomatic (16). As for symptomatic cases associated with disc herniation, surgery indication is considered. Our patient No.2 was installed in the knee-chest position and the other two in prone position. The choice of these two positions is related to ignorance of the existence of these anomalies and usual practices of services. Patient No.2 had had a discectomy by interlaminar appraoch, then a decompression of the lateral recessus and foraminal and the progression was well. Patient No.1 after an interlaminar approach received only foraminotomy without discectomy because due to the fact that the mobilization of the medial root was difficult. This conjoined roots mobilization according to Trimba (2012) is painful and could be responsible for inflammation post-operative neurologic deficit (7). The third patient received discectomy after a total laminectomy. A neurological iatrogenic deficiency has been reported often and consisted in an inadvertency portion incision of nerve anomaly taken as hernia protrusion $(13,17)$. Minimal invasive procedures increase operative failure incidence in a considerable way (11). Our three patients presented a favorable evolution after two years and two months for the first, one year and four months for the second and six months for the third.

\section{Conclusion}

This study is the first Senegalese series on the lumbosciatica by anomaly of root emergence and highlights especially the difficulties for the diagnosis of these anomalies like other sub-Saharan African countries where expansion of MRI for the diagnosis is low and still very expensive. MRI provides guidance signs and a large root appearance can warn about the existence of these anomalies. A good root release improves the symptoms.

\section{Funding}

None.

\section{Conflicts of Interest}

The authors declare that they have no conflicts of interest.

\section{References}

1. Lotan R, Al-Rashdi A, Yee A, Finkelstein J. Clinical features of conjoined lumbosacral nerve roots versus lumbar intervertebral disc herniations. Eur Spine J. 2010; 19: 1094-1098.

2. Zagnoni C. Reperto di un tipo non conosciuto di anastomosi nervosa delle radici spinali. Atti Soc Med Chir Padova 1949 27:48-52.

3. Taghipour M, Razmkon A, Hosseini K. Conjoined Lumbosacral Nerve Roots Analysis of Cases Diagnosed Intraoperatively. J Spinal Disord Tech. 2009; 22: 413-416.

4. White JG III, Strait TA, Binkley JR, Hunter SE. Surgical treatment of 63 cases of conjoined nerve roots. J Neurosurg 1982; 56: 114-7.

5. Postacchini F, Urso S, Ferro L. Lumbosacral nerve root anomalies. J Bone Joint Surg Am 1982; 64: 721-9.

6. Kadish LJ, Simmons EH. Anomalies of the lumbosacral nerve roots. An anatomical investigation and myelographic study. J Bone Joint Surg $\mathrm{Br}$ 1984; 66: 411-6.

7. Trimba R, Jeffrey M. Spivak, John A. Bendo. Conjoined nerve roots of the lumbar spine. The Spine Journal 12 (2012) 515-524.

8. Helms CA, Dorwart RH, Gray M. The CT appearance of conjoined nerve roots and differentiation from a herniated nucleus pulposus. Radiology 1982; 144: 803-7.

9. Phillips LH II, Sung PT. The frequency of intradural conjoined lumbosacral dorsal nerve roots found during selective dorsal rhizotomy. Neurosurgery 1993 ;31: 88-91

10. Scuderi GJ, Vaccaro AR, Brusovanik GV \& al. Conjoined lumbar nerve roots: a frequently underappreciated congenital abnormality. J Spinal Disord Tech. 2004; 17(2) :86-93.

11. Bouchard JM, Copty M, Langelier R. Preoperative diagnosis of conjoined roots anomaly with herniated lumbar discs. Surg Neurol 1978; 10: 229-31.

12. Decq P, Bokombe D, Nguyen Jp, Djindjian M, Molina P, Colin H, et al. Sciatique et anomalies d'émergence radiculaire : à propos d'une série de 20 cas. Neurochir. 1989; 35: 371-6.

13. Younes M, Korbaa W, Zrour S, Bejia I, Touzi M, Bergaoui N. Lombosciatique de causes inhabituelles: kystes de Tarlov et anomalies des racines nerveuses. Revue neurologique. 2009; 165: 282-287.

14. Kadish LJ, Simmons EH. Anomalies of the lumbosacral nerve roots. An anatomical investigation and myelographic study. J Bone Joint Surg Br 1984; 66: 411-6.

15. Artico M, Carloia S, Piacentini M et al. Conjoined lumbosacral nerve roots: observations on three cases and review of the literature. Neurocirugía 2006; 17 : 54-59.

16. Jeffrey LS, Richard AB, Robert EB and Richard HR. Surgical management of sciatica involving anomalous lumbar nerve roots. Journal of spine disorders 1988 1(2) 111-115.

17. Song SJ, Lee JW, Choi JY, et al. Imaging features suggestive of a conjoined nerve root on routine axial MRI. Skeletal Radiol 2008; 37: 133-8.

\section{Comments}

This is a well-written case series discussing the local experience with conjoined nerve root anomalies in sub-Saharan region. Although the number of cases is limited, the authors have provided a comprehensive discussion on the cases. These three cases provide a diverse collection of different scenarios a spine surgeon may encounter during surgery of a patient with conjoined nerve root anomaly. One patient was diagnosed in pre-operative MR imaging in an ideal story; another patient was intra-operatively proven to have one, later diagnosed retrospectively in a post-operative MRI; while the third patient suffered a redo surgery to find the anomaly as a cause of treatment failure. Unfortunately the majority of cases mimic the second or third scenario, and anomalies are routinely missed on pre-operative imaging studies $[1,2,3]$. The high cost of magnetic resonance imaging in poor countries is also an important limitation which may prevent a careful pre-operative diagnosis, which is carefully addressed in the study. Surgeon's clinical suspicion has been more effective than a radiologic diagnosis in at least less than half of cases [2]. Therefore, careful pre-operative neurological examination in addition to meticulous surgical technique are essential to provide optimal outcomes following surgery.

Ali Razmkon, MD, Assistant Professor of Neurosurgery, Department of Neurosurgery, Shiraz University of Medical Sciences, Shiraz, Iran

Mousa Taghipour, MD, Professor of Neurosurgery, Department of Neurosurgery, Shiraz University of Medical Sciences, Shiraz, Iran

\section{References}

1. Taghipour M, Razmkon A, Hosseini K. Conjoined Lumbosacral Nerve Roots; Analysis of Cases Diagnosed Intraoperatively. J Spinal Disord Tech. 2009; 22:413-416.

2. Lotan R, Al-Rashdi A, Yee A, Finkelstein J. Clinical features of conjoined lumbosacral nerve roots versus lumbar intervertebral disc herniations. Eur Spine J. 2010; 19: 10941098.

3. Trimba R, Jeffrey M. Spivak, John A. Bendo. Conjoined nerve roots of the lumbar spine. The Spine Journal. 2012; 12(6):515-524. 